Service de

Gastroentérologie, Hôpital Saint-Lazare

Paris, France

A Attar

M-C Boutron

B Flourié

Service de

Gastroentérologie, Hôpital Saint-Louis, Paris, France

M Lémann

Norgine Pharma, Paris, France M Halphen

Service de Gériatrie, Hôpital du Mans, France

E Alix

Service de Gastroentérologie, Hôpital de La Croix-Saint-Simon, Paris, France

M Salmeron

Service de

Gastroentérologie, Hôpital Claude Huriez, Lille, France

F Guillemot

Service de

Gastroentérologie, Hôpital Cochin, Paris, France

S Chaussade

Service de Gériatrie, Hôpital de

Villiers-Le-Bel, France

A-M Ménard

Service de

Gastroentérologie,

Hôpital Rangueil,

Toulouse, France

J Moreau

Service de

Gastroentérologie,

Hôpital des

Diaconnesses, Paris,

France

G Naudin

Service de

Gastroentérologie, Hôpital

Sainte-Marguerite, Marseille, France

M Barthet

Gastrointestinal Unit, Western General Hospital, Edinburgh, UK

A Ferguson

Correspondence to: Dr M Lémann, Service de Gastroentérologie, Hôpital Saint-Louis, 1 avenue Claude Vellefaux, 75010, Paris, France.

Accepted for publication 7 July 1998

\title{
Comparison of a low dose polyethylene glycol electrolyte solution with lactulose for treatment of chronic constipation
}

A Attar, M Lémann, A Ferguson, M Halphen, M-C Boutron, B Flourié, E Alix, M Salmeron, F Guillemot, S Chaussade, A-M Ménard, J Moreau, G Naudin, M Barthet

\begin{abstract}
Background-Polyethylene glycol (PEG) 3350 is a non-absorbable, nonmetabolised osmotic agent used in lavage solutions for gut cleansing.

Aims-To compare the efficacy of PEG and lactulose in chronic constipation.

Methods-A total of 115 patients with chronic constipation entered a multicentre, randomised, comparative trial. They initially received two sachets containing either PEG (13 g/sachet) or lactulose (10 g/sachet) and were given an option to change the dose to one or three sachets/ day, depending on response.
\end{abstract}

Results-Ninety nine patients completed the trial. After four weeks, patients in the PEG group $(n=50)$ had a higher number of stools and a lower median daily score for straining at stool than patients in the lactulose group $(n=49)$. Overall improvement was greater in the PEG group. Clinical tolerance was similar in the two groups, but flatus was less frequently reported in the PEG group. The mean number of liquid stools was higher in the PEG group but the difference was significant only for the first two weeks. There were no serious adverse events and no significant change in laboratory tests in either group. At the end of the study, the number of sachets used by the patients was $1.6(0.7) / d a y$ in the PEG group and 2.1 $(0.7) / d a y$ in the lactulose group. Sixty one patients completed a further two months open study of one to three sachets PEG daily; there was no loss of efficacy and no serious toxicity.

Conclusion-Low dose PEG 3350 was more effective than lactulose and better tolerated.

(Gut 1999;44:226-230)

Keywords: constipation; polyethylene glycol; lactulose; cathartics; randomised trial

Chronic constipation is a common symptom in gastroenterology and general practice. Standard treatment includes increasing the oral intake of fibre in the form of wheat bran or bulk forming agents. Although there is some evidence from controlled trials confirming their efficacy, ${ }^{12}$ these measures are often inadequate. They fail to normalise bowel habit in up to $40 \%$ of patients, and their use may be detrimental because of exacerbation of other symptoms such as abdominal pain, bloating, or flatulence. ${ }^{3}$ In patients unresponsive or intolerant to fibre, both osmotic and stimulant laxatives are effective, but the latter must be avoided for long term use as they have a potential for adverse effects. ${ }^{4}$ Among the osmotic agents, lactulose is a synthetic disaccharide that is not absorbed by the small intestine but is readily metabolised by colonic bacteria. It has been shown to be effective for treating constipation, especially in the elderly. ${ }^{5-8}$ Intracolonic fermentation of lactulose is associated with production of gases and with colic, bloating, and flatulence. Furthermore, chronic ingestion of lactulose may induce changes in colonic bacterial metabolism and reduced efficacy in the treatment of chronic constipation. ${ }^{9}$

Polyethylene glycol (PEG) 3350 is a mixture of non-absorbable, non-metabolised polymers of mean molecular weight $3350( \pm 10 \%)$ that act as pure osmotic agents. It contains no more than $0.1 \%$ of lower molecular weight PEGs that are absorbable either in healthy subjects or in patients with inflammatory bowel disease, and are excreted unchanged by glomerular filtration. ${ }^{10}$ PEG 3350 electrolyte solutions have been widely used for bowel cleansing before colonoscopy or bowel surgery. ${ }^{11}$ Even when large volumes are ingested, PEG induced diarrhoea is associated with minimal electrolyte losses or gains. ${ }^{11}{ }^{12}$ PEG 3350 electrolyte solutions are thus safer than osmotic salts such as magnesium and sodium sulphate or phosphate, especially in patients with impaired renal or cardiac function.

Recent data indicate that small volumes of PEG electrolyte solution may be effective for treatment of constipation. ${ }^{13-18}$ In a preliminary study conducted in nine healthy volunteers, ingestion of a solution containing PEG 3350 at doses of $26 \mathrm{~g} /$ day (two sachets of $13 \mathrm{~g}$ ) and 39 $\mathrm{g} /$ day (three sachets) for eight days significantly increased stool weight from $136 \mathrm{~g}$ during the control period to 225 and 288 g/day, respectively. ${ }^{19}$ The volume of water taken with PEG (30-190 ml) did not modify the results

As PEG is osmotically active without increased colonic gas production, we expected that it would be better tolerated than osmotic sugars such as lactulose. Thus, the aim of the present trial was to compare the efficacy, tolerance, and safety of a low daily dose of PEG 3350 electrolyte solution (13-39 g/day) with

Abbreviations used in this paper: PEG, polyethylene glycol. 
lactulose (10-30 g/day) in the treatment of chronic idiopathic constipation, over a one month period.

\section{Patients and methods \\ STUDY DESIGN}

The study was randomised with two parallel groups, and was carried out in 10 centres in France and Scotland. The randomisation was made using a computer algorithm. A randomisation list established by the statistician for each centre was provided to the manufacturer for preparation of the study drugs but the investigators were unaware of the allocation of treatments. Patients were treated with either PEG electrolyte solution or lactulose for four weeks. As the two treatments differed in appearance and taste, the study was not conducted double blind. The trial was performed in accordance with the principles stated in the Declaration of Helsinki and was approved by the local ethics committees. All the patients gave informed consent.

\section{PATIENTS}

Patients were recruited from both general and geriatric hospitals. The majority were outpatients from the departments of gastroenterology from nine hospitals. In order to assess the safety and acceptance of the drugs in elderly patients, we also recruited patients from three geriatric institutions, aiming for a ratio 70:30 general:geriatric patients. Patients of both sexes were considered for inclusion in the study if they were at least 18 years old and suffered from chronic idiopathic constipation. The diagnosis of chronic idiopathic constipation was based on the following criteria: (1) presence for at least three months of less than three stools per week and/or straining at stool; and (2) for patients older than 45 years, exclusion of constipation secondary to colonic disease, verified by a colonoscopy or a barium enema performed within the past five years. Patients taking concomitant medications which could modify bowel habit, and those suffering from severe liver, renal, or cardiac diseases were excluded, as were pregnant and breast feeding women. Patients previously exposed to lactulose were not excluded.

\section{STUDY DRUGS}

The investigational drug (Movicol, Norgine Pharma, Paris, France) was given as a powder in a sachet containing 13.12 g PEG 3350, 0.18 g sodium bicarbonate, $0.35 \mathrm{~g}$ sodium chloride, $0.05 \mathrm{~g}$ potassium chloride, $0.01 \mathrm{~g}$ acesulfame-K, and J 2076 lemon flavours. The reference drug was lactulose, given as a solution in a commercially available sachet containing $10 \mathrm{~g}$ lactulose diluted in $15 \mathrm{ml}$ water (Lactulose Biphar, Procter and Gamble Pharmaceuticals, France). The study drugs were packed in identical boxes and the drug the patient was receiving was not written on the packet. At entry in the study, patients were instructed to take two sachets per day of the medications, in two divided doses, each sachet being diluted in approximately $125 \mathrm{ml}$ of water. If stools became liquid, they could reduce the dosage to one sachet/day. After two weeks, patients were also given an option to change the dosage, and to take one, two, or three sachets/day, depending on the efficacy and their tolerance of the drug. No other treatments for constipation were allowed during the study, except for the use of suppositories or microenemas which were recorded on the daily diary card.

\section{EFFICACY AND TOLERANCE ASSESSMENT}

Clinical efficacy and tolerance were assessed using a daily diary card where the patient indicated the number of stools and the severity of the following symptoms: straining at stool, liquid stools, abdominal pain, bloating, flatus, and rumbling. For each symptom, a score ranging from 0 (absence) to 3 (severe) was used. At week 4, the overall improvement was assessed on a visual analogue scale, ranging from 0 (no change) to 10 (excellent). Laboratory blood tests including cell counts, electrolytes, glucose, urea nitrogen, protein, creatinine, folate, and vitamin $\mathrm{B}_{12}$ were performed at entry and after four weeks.

FOLLOW UP

After completion of the protocol, patients were given the opportunity to take PEG (one to three sachets/day) for two additional months, in order to evaluate the long term efficacy and safety of the treatment. Clinical efficacy and tolerance were assessed from the daily diary card. Laboratory tests were repeated at the end of the two month period.

\section{STATISTICAL ANALYSIS}

Based on previous experience, ,-8 $18^{18}$ we calculated that the number of patients to be included to show a reduction of digestive symptoms by $30 \%$ with PEG, compared with lactulose (about $50 \%$ in previous trials), was 58 patients per group (alpha risk $=0.05$; beta risk $=0.10$ ). For each patient the clinical efficacy and tolerance variables were analysed for the first two and the last two weeks, as well as for the four weeks of the study. Mean values were calculated for the number of stools, the number of sachets taken, and the overall improvement. The median daily score and the number of days the score was greater than 1 were calculated for the variables recorded on a 0-3 scale. Comparisons between the two treatment groups were performed using Student's $t$ tests or nonparametric Mann-Whitney $U$ tests according to the distribution of values and $\chi^{2}$ tests. Laboratory data were compared at entry and at week 4 using paired $t$ tests. Values were expressed as mean (SD); percentages were given with 95\% confidence intervals (CI). Values of $p$ less than 0.05 were considered significant.

\section{Results}

A total of 115 patients was allocated to treatment with either PEG $(n=60)$ or lactulose $(n=55)$. The number of patients included at each centre ranged from three to 24 . Thirty one patients $(27 \%)$ were recruited from geriatric institutions. The two treatment groups were well matched for all clinical characteristics (table 1). Sixteen patients (10 in the PEG 
Table 1 Patient characteristics

\begin{tabular}{lll}
\hline & PEG group & Lactulose group \\
\hline Number of patients & 60 & 55 \\
Age (years) & $55(24)$ & $55(22)$ \\
$\quad$ Age $\leqslant 65(\mathrm{n})$ & 35 & 37 \\
Age $>65(\mathrm{n})$ & 25 & 18 \\
Sex $(\mathrm{F} / \mathrm{M})$ & $51 / 9$ & $43 / 12$ \\
Inclusion criteria (n patients) & & \\
$\quad$ Less than 3 stools/week & 23 & 26 \\
$\quad$ Straining at stool & 6 & 8 \\
$\quad 31$ & 20 \\
Poth criteria & 10 & 6 \\
Premature withdrawal & 6 & 3 \\
$\quad$ Lost to follow up & 2 & 1 \\
Adverse effect & 2 & 2 \\
$\quad$ Treatment failure & & \\
\hline
\end{tabular}

group and six in the lactulose group) failed to complete the four week study satisfactorily and were excluded from the analysis because clinical efficacy and tolerance data at weeks 2 and 4 were not available. Table 1 gives the reasons for exclusion.

\section{EFFICACY}

Throughout the four week period, the mean stool frequency was higher in the PEG than in the lactulose group $(1.3(0.7)$ versus $0.9(0.6) /$ day; $p=0.005)$. The percentage of patients who passed less than three stools per week during treatment was however similar in the two treatment groups (10\% (95\% CI $1.5 \%$ to $18.5 \%$ ) and $14 \%$ (95\% CI $4.1 \%$ to $23.9 \%$ ) respectively). The median daily score for straining at stool was lower in the PEG group than in the lactulose group (0.5 (0.7) versus $1.2(0.9)$; $\mathrm{p}=0.0001)$; the number of days with a score greater than 1 was lower in the PEG group compared with the lactulose group (2.7 (5.4) versus 7.0 (5.7); $p=0.0001$ ). At the end of the study, the mean visual analogue scale ratings for overall improvement were 7.4 (2.5) in the PEG group and 5.2 (3.3) in the lactulose group $(p<0.001)$. The percentage of patients who used suppositories or microenemas at any time during the study was also significantly lower in the PEG group than in the lactulose group (16\% (95\% CI $6 \%$ to $26 \%)$ versus $34 \%$ (95\% CI $20 \%$ to $47 \%) ; \mathrm{p}=0.04)$. The results were similar when efficacy variables were compared between the two treatment groups for the first two or last two weeks of the trial.

\section{TOLERANCE}

There were no significant differences between the two treatment groups with respect to median daily scores of clinical symptoms - that is, liquid stools $(0.3(0.9)$ in the PEG group versus $0.2(0.7)$ in the lactulose group, respectively), abdominal pain (0.4 (0.8) versus 0.7 $(0.9))$, bloating $(0.7(0.9)$ versus $0.9(1.0))$, flatus $(0.8(0.8)$ versus $1.0(0.9))$, and rumbling $(0.2(0.5)$ versus $0.4(0.8))$. The number of days with a score greater than 1 was similar in the two treatment groups for liquid stools (4.1 (5.5) versus 2.1 (4.6)), bloating (5.6 (8.4) versus 8.9 (10.3)), and rumbling (2.4 (5.4) versus $4.1(7.7))$; it was significantly lower in the PEG group for flatus (3.8 (6.8) versus $9.2(10.1) ; \mathrm{p}=0.01)$ and there was a trend in favour of PEG for abdominal pain (3.9 (6.6) versus $6.8(9.0) ; p=0.08)$. Figure 1 shows

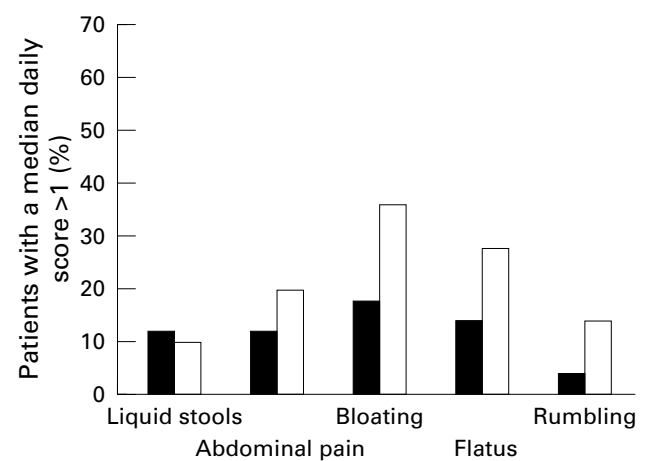

Figure 1 Clinical tolerance of PEG (black bars) and lactulose (white bars). The percentage of patients with a median daily score greater than 1 was significantly lower in the PEG group for bloating $(p<0.05)$ and a trend was found for abdominal pain, flatus, and rumbling.

the percentages of patients with a median daily score greater than 1 . The results were similar when the symptomatic scores were compared between the two treatment groups for the first two and the last two weeks of the trial. For the four week period, the mean number of liquid stools was higher in the PEG group than in the lactulose group (2.4 (3.5) versus 0.6 (1.2), $\mathrm{p}=0.001)$ but the difference was significant only for the first two weeks of the trial (2.9 (4.3) versus $0.5(1.0) ; \mathrm{p}<0.001)$.

Three adverse events leading to drug withdrawal were recorded during the study, two in the PEG group (acute diarrhoea with vomiting and fever in one patient, abdominal pain in another patient) and one in the lactulose group (depression). No significant change in mean laboratory assessments was noted during the study within both treatment groups, with the exception of a slight but significant decrease in sodium concentration in the lactulose group (140 (2.2) at entry versus $139(3.0) \mathrm{mmol} / \mathrm{l}$ at four weeks; $\mathrm{p}=0.02)$. A mild hypokalaemia was noted in two patients who concurrently received diuretics, one in each treatment group.

\section{TREATMENT DOSAGE}

The mean number of sachets used every day during the first two weeks of the study did not differ significantly between the two treatment groups (1.8 (0.4) sachets/day in the PEG group versus 1.9 (0.5) in the lactulose group). During the last two weeks of the study, when the patients were free to adjust the daily dose, the number of sachets used per day was $1.6(0.7)$ in the PEG group and 2.1 (0.7) in the lactulose group $(p<0.001)$. Figure 2 shows the percentage of patients taking the different dosages of the two medications during the last two weeks of the study.

FOLLOW UP

Of the 99 patients who completed the four week protocol, 65 were treated on an open basis with PEG, and 61 of these completed two additional months' follow up. The mean number of sachets used by these patients throughout the two month period was $1.5(0.6)$ per day. The frequency of stools was $1.3(0.7)$ per day. The median daily scores at the 


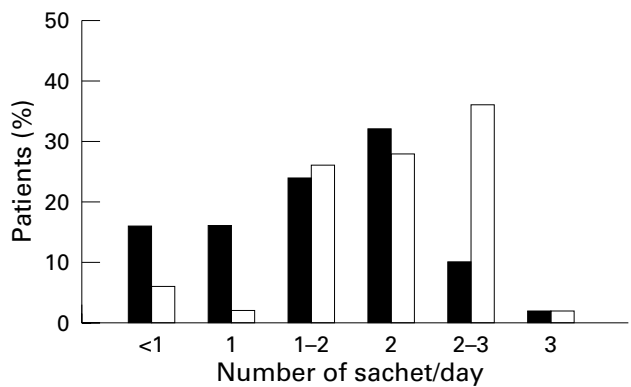

Figure 2 Percentage of patients taking the different dosages of PEG (black bars) and lactulose (white bars) during the last two weeks of the study.

beginning and end of the two month period were similar for all clinical symptoms. There were no significant changes in laboratory test results, with the exception of the mean serum folate level which was significantly lower after three months compared with the value at four weeks (6.0 (3.3) versus $7.1(4.4) \mu \mathrm{g} / \mathrm{l} ; \mathrm{p}=0.01)$. However, the individual values of serum folate remained in the normal range for all patients. Four adverse events leading to drug withdrawal were recorded: acute diarrhoea with fever in one patient, abdominal pain in two patients, and vomiting in one patient.

\section{Discussion}

In this study, PEG 3350 given at a low daily dose (13-39 g/day) was more effective than lactulose (10-30 g/day) for the treatment of chronic constipation. Stool frequency was higher and the median daily score for straining at stool was lower in the PEG group. The percentage of patients who used suppositories or microenemas was also significantly lower in the PEG group. As the two treatments differed in appearance and taste, our study could not be conducted double blind. We cannot exclude the possibility that some biases may have arisen because of this methodological limitation, in particular for subjective efficacy assessment. However, PEG was superior to lactulose for all the efficacy variables, including the number of stools which can be regarded as the more objective efficacy variable.

It should be noted that a few of the patients have experienced some degree of PEG induced diarrhoea. The number of liquid stools was however small, and the difference between the two treatment groups was significant only for the first two weeks of the trial, when the patients had been asked to initiate the treatment with a fixed dose of two sachets of medication per day. The starting dosage of PEG had been chosen on the basis of results in a study of healthy volunteers which showed that 26-39g PEG per day significantly increases the mean daily stool weight. ${ }^{19}$ During the last two weeks of the present trial, patients were given an option to select a dosage from one to three sachets/day, depending on the efficacy and tolerance of the drug. Only $36 \%$ of patients from the lactulose group used a dose lower than two sachets per day during the last two weeks of the study, while $38 \%$ used a higher dose. In contrast, a majority of the patients $(56 \%)$ in the PEG group used a lower daily dose of medication, while only $12 \%$ used a higher dose. Thus, we recommend that in future, treatment with PEG should be initiated using one sachet per day, and the dose increased to two or three sachets per day if required.

PEG and lactulose are usually classified as osmotic agents. In our study, the osmotic loads of the two treatment were not equivalent: for lactulose, the ingested osmotic load corresponding to $20 \mathrm{~g}$ of medication is approximately $60 \mathrm{mmol} /$ day, compared with $20 \mathrm{mmol} /$ day for $26 \mathrm{~g}$ of PEG. However, at the dose used in our patients, the laxative effect of lactulose is not directly due to an osmotic effect of the non-absorbed sugar. ${ }^{10}$ The intracolonic osmotic load due to unabsorbed organic acids produced by colonic bacterial metabolism of lactulose is unknown.

The main objective of our study was to compare the clinical tolerance of PEG and lactulose. As lactulose is metabolised by the colonic bacterial flora to produce short chain fatty acids, one would expect that its laxative effect would be associated with a notable production of gases including carbon dioxide, methane, and hydrogen. Most of the common side effects attributed to lactulose such as abdominal pain, bloating, and flatus are thought to result from this colonic fermentation. In our study, however, there were no significant differences between the two treatment groups with respect to median daily scores of clinical symptoms including abdominal pain, bloating, flatus, and rumbling. The number of days with a score greater than 1 were also similar in the two treatment groups, with the exception of flatus which was significantly more frequent in the lactulose group; a trend was also found for abdominal pain. Additionally, the percentage of patients with a median daily score greater than 1 was greater in the lactulose group for bloating and a trend was found for abdominal pain, flatus, and rumbling.

In previous placebo controlled studies evaluating PEG in the treatment of chronic constipation, similar symptoms were also reported by the patients, ${ }^{17}{ }^{18}$ but at the same frequency in PEG and placebo groups, apart from rumbling that occurred more frequently with PEG in one study. ${ }^{18}$ As PEG is not metabolised by the colonic flora, it can be inferred from our results that most of the symptoms reported by the patients and attributed to intracolonic metabolism of lactulose $e^{5-8}$ were actually partly related to colonic fermentation. As these symptoms are equally reported by patients from the PEG and lactulose groups, it is likely that they are partly due to irritable bowel syndrome, a condition which may be associated with chronic idiopathic constipation.

The present study also confirms that low dose PEG, when given for three months, is safe. No serious side effects related to the drug were observed, in accordance with the safety profile in previous trials. ${ }^{13-18}$ Chronic toxicity studies have been carried out in animals with PEG 1540 and 4000 being mixed in the food over a two year period without any signs of 
toxicity. ${ }^{20}$ Furthermore, there is now experience with the drug in a large number of patients in countries where low dose PEG is marketed. In the present study, there were no significant changes in blood tests performed at entry and after one to three months of PEG, with the exception of a slight but statistically significant decrease in the mean serum folate level in the subgroup of patients who were treated with PEG for three months. However, individual values always remained in the normal range. As our patients were partly recruited in geriatric institutions, $37 \%$ of them ranged in age from 65 to 89 years. PEG was well tolerated in this subgroup of elderly patients.

In conclusion, the present study shows that PEG 3350 at low daily doses (13-39 g/day) was more effective and better tolerated than lactulose (10-30 g/day) in the treatment of chronic idiopathic constipation. Low dose PEG may be regarded as a good alternative in patients with constipation refractory to more conventional and less costly treatments. There was no serious toxicity of PEG after one to three months of treatment. The optimal dosage was between 13 and $26 \mathrm{~g}$ of PEG per day in most of the patients.

1 Badiali D, Corazziari E, Habib FI, et al. Effect of wheat bran in treatment of chronic nonorganic constipation. A doublein treatment of chronic nonorganic constipation. A
blind controlled trial. Dig Dis Sci 1995;40:349-56.

2 Graham DY, Moser SE, Estes MK. The effect of bran on braham DY, Moser SE, Estes MK. The effect of bran on bowel functi

3 Francis CY, Whorwell PJ. Bran and irritable bowel syndrome: time of reappraisal. Lancet 1994;344:39-40

4 Thompson WG. Laxatives: clinical pharmacology and practical use. Drugs 1980;19:49-58.

5 Lederle FA, Busch DL, Mattox KM, et al. Cost-effective treatment of constipation in the elderly: a randomized double-blind comparison of sorbitol and lactulose. $A m 7$ Med 1990;89:597-601.

6 Sanders JF. Lactulose syrup assessed in a double-blind study of elderly constipated patients. F Am Geriatr Soc 1978;26:236-9.

7 Passmore AP, Wilson-Davies K, Stoker C, et al. Chronic constipation in long-stay elderly patients: a comparison of lactulose and a senna-fibre combination. BMF 1993;307: 769-71.

8 Wesselius-De Casparis S, Braadbaart S, van der BerghBohlken GE, et al. Treatment of chronic constipation with lactulose syrup: results of a double-blind study. Gut 1968; 9:84-6.

9 Florent C, Flourié B, Leblond A, et al. Influence of chronic lactulose ingestion on the colonic metabolism of lactulose in man (an in vivo study). $\mathcal{F}$ Clin Invest 1985;75:608-13.

10 Berry MA, DiPalma JA. Review article: orthograde gut lavage for colonoscopy. Aliment Pharmacol Ther 1994;8:391-5.

11 Hammer HF, Santa Ana CA, Schiller LR, et al. Studies of osmotic diarrhea induced in normal subjects by ingestion of polyethylene glycol and lactulose. F Clin Invest 1989;84: 1056-62.

12 Brady III CE, DiPalma JA, Morawski SG, et al. Urinary excretion of polyethylene glycol electrolyte lavage solution. Gastroenterology 1986;90:1914-18.

13 Andorsky RI, Goldner F. Colonic lavage solution (polyethylene glycol electrolyte lavage solution) as a treatment for chronic constipation: a double-blind, placebo-controlled study. Am 7 Gastroenterol 1990;85:261-5.

14 Puxty AH, Fox RA. Golytely: a new approach to fecal impaction. Age Aging 1986;15:182-4.

15 Tolia V, Lin CH, Elitsur Y. A prospective randomized study with mineral oil and oral lavage solution for treatment of faecal impaction in children. Aliment Pharmacol Ther 1993; 7:523-9.

16 Klauser AG, Mühldorfer BE, Voderholzer WA, et al. Polyethylene glycol 4000 for slow transit constipation. $Z$ Gastroenterol 1995:33:5-8.

17 Corazziari E, Badiali D, Habib FI, et al. Small volume isomotic polyethylene glycol electrolyte balanced solution (PMF-100) in treatment of chronic nonorganic constipation. Dig Dis Sci 1996;41:1636-42.

18 Lémann M, Chaussade S, Halphen M, et al. Low dose polyethylene glycol in chronic constipation: double-blind placebo-controlled crossover trial [abstract]. Gastroenterology 1996;110:A704.

19 Flourié B, Halphen M, Lémann M, et al. Effets digestifs de faibles doses de polyethylène glycol chez le sujet sain [abstract]. Gastroenterol Clin Biol 1994;18:A108.

20 Smyth HF, Carpenter CP, Weil CS. The chronic oral toxicology of the polyethylene glycols. $f$ Am Pharm Assoc Sci 1955;44:27-31. 